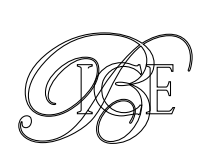

Iván Santos Esteras*

\title{
ESTADO DE LA LIBERALIZACIÓN DE LOS SERVICIOS DE TRANSPORTE DE PASAJEROS POR FERROCARRIL
}

La aprobación del Cuarto Paquete Ferroviario en 2016 supuso la culminación del paulatino proceso de liberalización de los mercados ferroviarios en Europa, con la apertura a la competencia de los servicios de transporte de viajeros por ferrocarril el 14 de diciembre de 2020. En abril de 2019, ADIF Alta Velocidad publicó la capacidad disponible en los tres principales corredores de alta velocidad, que se adjudicaría a los candidatos que se comprometieran a un mayor uso en un periodo de diez años. En este artículo se analiza esta regla de asignación, que ha sido objeto de discusión por su novedoso enfoque, y los efectos que ha supuesto en el proceso de liberalización.

Palabras clave: sector ferroviario, liberalización, ADIF.

Clasificación JEL: L40, R41.

\section{Introducción}

La aprobación del Cuarto Paquete Ferroviario en 2016, tras un largo proceso de negociación entre Comisión Europea, Consejo y Parlamento, supuso la culminación del paulatino proceso de liberalización de los mercados ferroviarios en Europa que había comenzado en el año 2001. De esta forma, se impuso la apertura a la competencia de los servicios de transporte de viajeros por ferrocarril el 14 de diciembre de 2020.

La transposición de esta disposición al ordenamiento jurídico español, en diciembre de

\footnotetext{
* Subdirector del Sector Ferroviario. Comisión Nacional de los Mercados y la Competencia (CNMC).

Versión de julio de 2020.

DOI: https:/doi.org/10.32796/bice.2020.3127.7084
}

2018, inició el proceso de liberalización de un sector caracterizado por importantes barreras a la entrada. En particular, en abril de 2019, los administradores de infraestructuras publicaron una oferta de capacidad marco, dando transparencia a la capacidad disponible para las empresas ferroviarias potencialmente interesadas en participar en este proceso. ADIF Alta Velocidad optó por un sistema de asignación que priorizaba las solicitudes de capacidad que aseguraran un uso intensivo de los tres corredores de mayor tráfico en la red de alta velocidad: Madrid-Barcelona, Madrid-Valencia/Alicante y Madrid-Sevilla/Málaga.

Seis empresas participaron en el proceso, siendo adjudicatarias las tres que solicitaron un porcentaje elevado de la capacidad puesta a disposición. Este resultado hace prever un $\triangleright$ 
importante crecimiento de la actividad ferroviaria una vez se desplieguen completamente los planes de negocio de los candidatos. El diseño del proceso de adjudicación ha sido objeto de discusión y análisis dado el enfoque novedoso que suponía y el papel jugado en el mismo por el gestor de infraestructuras.

El objeto de este artículo es describir, en primer lugar, la evolución normativa tanto a nivel europeo como español que culmina con la apertura de los servicios de transporte nacional de viajeros. En segundo lugar, se detallará el proceso seguido por ADIF Alta Velocidad para implementar el acceso de nuevos entrantes a la red ferroviaria y, en particular, el modelo diseñado para asignar la capacidad. A este respecto se analizarán las posibles alternativas con que contaba el gestor de infraestructuras para el diseño del proceso de asignación. Finalmente se señalarán otros elementos esenciales para el éxito de la liberalización, como el acceso a las estaciones de viajeros, y su estado actual.

\section{Antecedentes}

\subsection{Marco europeo}

Como se ha mencionado anteriormente, la liberalización de los servicios ferroviarios ha sido un largo proceso impulsado desde la Unión Europea. El denominado Cuarto Paquete Ferroviario fija el horizonte para su conclusión con la apertura de los mercados nacionales de transporte de pasajeros.

La progresiva introducción de la competencia en el sector ferroviario se inició con la aprobación del Primer Paquete Ferroviario (2001) ${ }^{1}$,

Directiva 2001/12/CE, Directiva 2001/13/CE, Directiva 2001/24/CE, Directiva 2001/16/CE. que introdujo la independencia en la gestión del administrador de infraestructuras con respecto a la prestación de los servicios y liberalizó los servicios internacionales de transporte de mercancías, garantizando los derechos de acceso a la red transeuropea a todos los operadores ferroviarios autorizados, de forma transparente y no discriminatoria.

El Segundo Paquete Ferroviario $(2004)^{2}$ se centró en medidas relativas a la seguridad y la interoperabilidad, y en la creación de la Agencia Ferroviaria Europea (ERA) ${ }^{3}$, encargada de dirigir la labor técnica en materia de seguridad e interoperabilidad. Este paquete, además, estableció la apertura total de los mercados ferroviarios de mercancías el 1 de enero de 2007.

El Tercer Paquete Ferroviario $(2007)^{4}$ se orientó hacia la liberalización del transporte internacional de viajeros, estableciendo el 1 de enero de 2010 como fecha límite para su apertura. Este Tercer Paquete persigue promover la constitución del mercado interior de los servicios ferroviarios mediante un marco estricto de normas de seguridad y la protección de los derechos de los viajeros.

En 2012 se aprobó la Directiva 2012/34/UE que, además de consolidar los diferentes paquetes ferroviarios en relación con la apertura del mercado, introdujo nuevas normas relativas a los cánones de acceso a la red ferroviaria, obligaciones sobre los explotadores de instalaciones de servicio y reforzó las competencias de los reguladores nacionales.

Así las cosas, el 30 de enero de 2013, la Comisión Europea analizó en profundidad el $\triangleright$

\footnotetext{
2 Directiva 2004/49/CE, Directiva 2004/50/CE, y Directiva 2004/51/ CE.

3 ERA (European Union Agency for Railways) es la agencia ferroviaria europea cuya creación se aprobó en el Reglamento (CE) 881/2004 del Parlamento Europeo y el Consejo.

4 Directiva 2007/58/EC, Directiva 2007/59/EC, Reglamento 2007/2370/CE, Reglamento 2007/1371/CE.
} 
desarrollo del sector ferroviario en Europa ${ }^{5}$, señalando que: i) se mantenía la segmentación del espacio ferroviario europeo, con una importante carga para las empresas ferroviarias, incluyendo más de 11.000 normas nacionales, costes de homologación del material rodante, etc.; ii) se mantenía el conflicto de interés de los administradores de infraestructuras integrados con las empresas prestadoras de servicios ferroviarios, generando situaciones discriminatorias para los nuevos entrantes; iii) la cuota modal del transporte de pasajeros por ferrocarril se había reducido en los últimos años, a pesar de las inversiones realizadas.

Por ello, la Comisión Europea propuso un amplio paquete normativo, denominado Cuarto Paquete Ferroviario, con el objetivo de impulsar el transporte por ferrocarril y hacerlo más competitivo. Este paquete ferroviario está formado por dos pilares: el pilar técnico ${ }^{6}$ y el pilar de mercado?.

El pilar de mercado completa el proceso de apertura gradual de los mercados, iniciado con el Primer Paquete Ferroviario, con la apertura del mercado del transporte nacional de viajeros, y otorga a las empresas ferroviarias el derecho de acceso a las infraestructuras ferroviarias de todos los Estados miembros en $2020^{\circ}$. Además, este pilar establece reglas para mejorar la imparcialidad en la gobernanza de las infraestructuras ferroviarias, evitando comportamientos discriminatorios, e introduce la licitación obligatoria para los contratos de Obligaciones de Servicio Público (OSP) de ferrocarril a partir

5 https://eur-lex.europa.eu/legal-content/ES/TXT/PDF/?uri=CELEX: $52013 D C 0025 \&$ from $=E S$

6 Directiva 2016/797/UE Interoperabilidad, Directiva 2016/798/UE Seguridad, Reglamento (UE) 2016/796 ERA.

7 Directiva 2016/2370/UE, Reglamento (UE) 2016/2337, Reglamento (UE) 2016/2338.

8 La Directiva 2016/2370/UE determina que la efectividad de este derecho se aplicará el 1 de enero de 2019, a tiempo para el horario de servicio de 14 de diciembre de 2020. del año 2023. Las medidas contenidas en el pilar de mercado tienen como objetivo final aumentar las posibilidades de elección para los usuarios y conseguir una mayor calidad en los servicios de transporte de viajeros por ferrocarril.

\subsection{Transposición al ordenamiento jurídico español}

En España, el proceso de liberalización se inicia con la aprobación de la Ley 39/2003, de 17 de noviembre, del Sector Ferroviario ${ }^{9}$, que entró en vigor el 1 de enero de 2005 y liberalizó el transporte de mercancías por ferrocarril ${ }^{10}$.

Esta ley creó el Comité de Regulación Ferroviaria (CRF), organismo regulador en España con funciones en la supervisión y resolución de conflictos entre el administrador de infraestructuras ferroviarias y los operadores del mercado ferroviario, o de estos entre sí, y para garantizar el correcto funcionamiento general del sistema ferroviario. La Ley $3 / 2013$, de 4 de junio, de creación de la Comisión Nacional de los Mercados y la Competencia suprimió el CRF y atribuyó sus funciones al nuevo organismo regulador.

La Ley 15/2009, de 11 de noviembre, del contrato de transporte terrestre de mercancías, liberalizó los servicios de transporte internacional de pasajeros por ferrocarril a partir del 1 de enero de 2010. Sin embargo, hasta la aprobación del Cuarto Paquete Ferroviario y las perspectivas de liberalización de los servicios nacionales este segmento del mercado no interesó a las empresas ferroviarias alternativas. Tras $\triangleright$

\footnotetext{
9 Esta ley incorporó al ordenamiento jurídico español las normas comunitarias que integran el Primer Paquete Ferroviario.

10 En España, la liberalización del transporte de mercancías se produjo dos años antes de la fecha del 1 de enero de 2007, establecida en el Segundo Paquete Ferroviario.
} 
su aprobación, Intermodalidad del Levante propuso un nuevo servicio entre Madrid y Montpellier y Arriva Spain Rail entre A Coruña y Oporto.

Posteriormente, el Real Decreto Ley 4/2013, de 22 de febrero, de medidas de apoyo al emprendedor y de estímulo del crecimiento y de la creación de empleo continuó el proceso de apertura de los servicios de transporte de viajeros en España, liberalizando, a partir del 31 de julio de 2013, los servicios de transporte con finalidad prioritariamente turística ${ }^{11}$.

Este real decreto ley también previó la apertura de los servicios de transporte nacional de viajeros por ferrocarril mediante la obtención de títulos habilitantes en líneas o corredores establecidos mediante Acuerdo del Consejo de Ministros. El Acuerdo de 13 de junio de 2014 estableció la posibilidad de otorgar un segundo título habilitante para el transporte en el corredor del Levante. Sin embargo, este proceso quedó superado por la inminente aprobación del señalado Cuarto Paquete Ferroviario.

La Ley 38/2015, de 29 de septiembre, del Sector Ferroviario (Ley del Sector Ferroviario) incorporó, si bien parcialmente ${ }^{12}$, al ordenamiento jurídico español la Directiva 2012/34/ UE, que establece el espacio ferroviario único europeo.

Por último, el Real Decreto Ley 23/2018 modificó la Ley 38/2015 para avanzar en la transposición de la citada Directiva 2012/34/UE e incorporar la Directiva (UE) 2016/2370, que

\footnotetext{
11 Estos servicios se definen como aquellos en los que la totalidad de las plazas ofertadas en el tren se prestan en el marco de una combinación previa, vendida $u$ ofrecida en venta por una agencia de viajes con arreglo a un precio global en el que, aparte del servicio de transporte ferroviario, se incluyan, como principales, servicios para satisfacer de una manera general las necesidades de las personas que realizan desplazamientos relacionados con actividades recreativas, culturales o de ocio, siendo el servicio de transporte por ferrocarril complemento de los anteriores.

12 La Comisión Europea ha abierto dos procedimientos de infracción en relación con la transposición de la Directiva 2012/34/UE en España:

https://ec.europa.eu/commission/presscorner/detail/en/MEMO_18_3446

https://ec.europa.eu/commission/presscorner/detail/en/inf_20_859
}

desarrolla el espacio único europeo. Este real decreto ley, además de reforzar la independencia e imparcialidad de los gestores de infraestructuras, estableció la apertura de los servicios de transporte de viajeros por ferrocarril el 14 de diciembre de 2020.

\section{Inicio del proceso: acceso a la red ferroviaria}

\subsection{Oferta de capacidad marco}

Propuesta de modificación de la Declaración sobre la Red

La aprobación del Real Decreto Ley 4/2013 y el establecimiento definitivo de la fecha definitiva de la liberalización de los servicios de transporte nacional de pasajeros por ferrocarril despertó el interés por el mercado español de alta velocidad de varios grupos empresariales. Esta situación es inédita en los procesos de apertura en Europa, en los que la competencia se había producido mayoritariamente entre la empresa histórica y un operador alternativo.

Por otra parte, si bien la red española de alta velocidad presenta índices reducidos de utilización, la capacidad de las principales estaciones de viajeros supone un cuello de botella que limita el uso potencial de la totalidad de la red.

Las cuantiosas inversiones necesarias para operar los servicios de transporte de viajeros de alta velocidad, así como las potenciales restricciones a la capacidad disponible, señalaron la necesidad de que el gestor de infraestructuras concluyera acuerdos marco a largo plazo con las empresas ferroviarias. Los acuerdos marco, un instrumento inédito en España, $\triangleright$ 
pero ampliamente utilizado en otros países europeos con redes ferroviarias con restricciones de capacidad, asegura a las empresas ferroviarias una determinada capacidad en la red, si bien no concreta las características específicas que deben establecerse en el proceso anual de asignación de capacidad.

Los acuerdos marco están regulados en el Reglamento de la Comisión Europea 2016/545 ${ }^{13}$, que establece que el gestor de infraestructuras puede publicar una oferta de capacidad marco, en la que se dé transparencia a la capacidad total puesta a disposición de terceros, los acuerdos marco suscritos y la disponibilidad para suscribir nuevos acuerdos. Alternativamente, estos pueden optar por esperar a que las empresas ferroviarias soliciten capacidad marco. En este caso, el administrador deberá informar al resto de empresas ferroviarias y candidatos, que, en su caso, podrán mostrar su interés por la misma.

A mediados de abril de 2019, ADIF Alta Velocidad sometió a consulta pública una modificación de la Declaración sobre la Red ${ }^{14}$, en la que propuso su oferta de capacidad marco en los tres principales corredores de alta velocidad en España: i) Madrid-Barcelona; ii) Madrid-Valencia/Alicante; y iii) Madrid-Sevilla/ Málaga.

La oferta de capacidad marco vinculaba los surcos en las líneas ferroviarias a la capacidad en las estaciones, que, como se ha señalado anteriormente, son cuellos de botella que limitan el uso potencial del conjunto de la red. De esta forma, ADIF Alta Velocidad aseguraba la

\footnotetext{
13 Reglamento de Ejecución (UE) 2016/545 de la Comisión de 7 de abril de 2016 sobre los procedimientos y criterios relativos a los acuerdos marco de adjudicación de capacidad de infraestructura ferroviaria.

14 La Declaración sobre la Red es un documento que debe elaborar el administrador de infraestructuras en el que debe detallar las características de la red ferroviaria que gestiona, las condiciones de acceso, incluyendo las económicas, y los servicios que se prestan. Este documento debe estar disponible de forma gratuita y en al menos dos idiomas.
}

capacidad en la línea y las estaciones, con tiempos de rotación de 45 minutos, asegurando una utilización eficiente del material rodante.

Además de dar transparencia a la capacidad disponible, ADIF Alta Velocidad la estructuró en tres paquetes que incluían surcos en los tres corredores señalados anteriormente. El paquete A suponía en torno al $65 \%$ de la capacidad marco total; el B, el 30\%; y el C, el 5\%. El paquete $A$ suponía un ligero incremento de los surcos con respecto a los servicios prestados hasta ese momento por Renfe, mientras que el paquete $B$ permitía un modelo de negocio que compitiera con el anterior en términos de frecuencias. Finalmente, el paquete $\mathrm{C}$ podría corresponderse con la entrada de un operador de nicho, encaminado a una competencia esencialmente en precios, dadas sus escasas frecuencias.

Estos paquetes de capacidad se adjudicarían a las empresas ferroviarias que se comprometieran con un mayor uso durante un periodo de diez años. ADIF Alta Velocidad se reservaba la posibilidad de no adjudicar la capacidad marco en caso de que no se alcanzara un aprovechamiento mínimo del $65 \%$ de los surcos incluidos en cada paquete.

\section{Visión de la CNMC}

La CNMC aprobó un informe sobre esta propuesta el 25 de junio de $2019^{15}$ en el que se valoraba positivamente la iniciativa del gestor, dado que permitía conocer la disponibilidad de capacidad en la red a las empresas ferroviarias potencialmente entrantes al mercado. La $\triangleright$

\footnotetext{
15 Acuerdo por el que se emite informe relativo a las propuestas de ADIF y ADIF Alta Velocidad sobre la modificación de la declaración sobre la red de 2019.

https://www.cnmc.es/sites/default/files/2541816_2.pdf
} 
capacidad marco ofertada permitía un resultado competitivo en el que convivieran al menos tres operadores con cuotas de mercado potencialmente superiores a las alcanzadas por las empresas alternativas en mercados abiertos a la competencia, como Italia o Austria ${ }^{16}$.

Sin embargo, la CNMC, reconociendo las competencias que el marco regulador otorga a los gestores de infraestructuras para optimizar el uso de la red, también señaló que la oferta de capacidad marco adolecía de una excesiva rigidez, lo que restringía excesivamente la libertad de los candidatos para implementar sus modelos de negocio. La adjudicación de capacidad es un proceso regulado que asegura el derecho de las empresas ferroviarias, y los candidatos deben poder solicitar aquellos surcos y corredores que más se ajusten a su propuesta comercial. Por el contrario, el diseño propuesto por ADIF Alta Velocidad se asemejaba más a una licitación pública en el que el gestor determinaba de forma excesiva las peticiones y el resultado del proceso.

Por ello, el Informe de la CNMC señaló que se debía flexibilizar la oferta de capacidad marco permitiendo peticiones que no estuvieran vinculadas a ninguno de los paquetes en los que se estructuraba la oferta de capacidad. Además, los candidatos debían poder solicitar la capacidad en los corredores que consideraran adecuados, por lo que debía eliminarse el límite mínimo de aprovechamiento del 65\%. Así, en caso de contar con capacidad, el gestor debía atender todas las solicitudes recibidas.

El interés que había despertado la liberalización del mercado en España podía derivar, como de hecho sucedió, en una sobredemanda que superara la capacidad disponible. Para

\footnotetext{
16 De acuerdo con el 6.ำ Informe de la Comisión Europea sobre el desarrollo del mercado ferroviario, NTV (Italia) y WESTbahn (Austria), que entraron entre 2011 y 2012, contarían con una cuota en torno al $25 \%$ y al $20 \%$ respectivamente.
}

esta situación debía preverse, en primer lugar, un proceso de coordinación que intentara satisfacer todas las peticiones. Sin embargo, la coordinación no podía suponer reducciones que desnaturalizaran los modelos de negocio de los candidatos, por lo que, además, debían establecerse criterios de priorización.

La CNMC concluyó que, en una situación de exceso de solicitudes que no pudieran coordinarse, el aprovechamiento de los surcos era un criterio transparente y no discriminatorio para asignar la capacidad marco. El periodo de diez años para considerar las peticiones también resultaba adecuado para equilibrar las situaciones de partida de candidatos con material rodante disponible y otros que debían adquirirlo, lo que retrasaba su fecha de entrada al mercado. Sin embargo, de nuevo, este horizonte no podía entenderse como vinculante, de forma que los candidatos pudieron solicitar capacidad en los ejercicios que consideraron adecuados.

Finalmente, dada la estructura del tráfico de viajeros en España y la importancia del corredor entre Madrid y Barcelona, priorizar el aprovechamiento de todos los corredores también se valoró positivamente como instrumento del gestor para optimizar el uso de la red y extender los beneficios de la competencia a un número mayor de usuarios.

Por tanto, cuando fuera posible, el gestor de infraestructuras debía intentar coordinar y atender todas las peticiones recibidas. Únicamente cuando la demanda de capacidad marco superara la capacidad disponible se aplicarían los criterios de priorización establecidos por ADIF Alta Velocidad, esto es, el mayor aprovechamiento de los surcos ofertados en el plazo de 10 años.

En este caso, la CNMC también señaló que, de priorizar solicitudes, un candidato únicamente podría ser adjudicatario de un paquete de capacidad marco. Además, dichos $\triangleright$ 
paquetes debían ser asignados a candidatos independientes con el objetivo de maximizar el número de empresas compitiendo en el mercado de transporte de viajeros por ferrocarril.

\subsection{Adjudicación de la capacidad marco}

\section{Proceso desarrollado por ADIF AV}

El 22 de julio de 2019, ADIF Alta Velocidad publicó la versión definitiva de la modificación de la Declaración sobre la Red 2019, en la que se introdujeron cambios con respecto a la versión inicial. Así, el gestor de infraestructuras eliminó el límite de aprovechamiento mínimo de la capacidad marco para su adjudicación, señalando que, si las solicitudes de capacidad fueran compatibles, todas ellas serían atendidas con el objetivo de maximizar el uso de la red y la entrada de nuevos operadores. Además, se establecía que los candidatos podrían solicitar capacidad de todos los paquetes, pudiendo realizar las solicitudes de capacidad marco que consideraran más adecuadas a su plan comercial, no estando vinculados a solicitar ni todos los servicios ni todos los ejes dentro de un paquete.

En cuanto a los criterios de adjudicación, la Declaración sobre la Red indicó que cuando las solicitudes de capacidad fueran incompatibles se daría prioridad a empresas diferentes, verificándose que no forman parte del mismo grupo empresarial, ni sus socios ostentan participaciones de control o ejercen influencia decisiva en otros adjudicatarios de capacidad. Además, ADIF Alta Velocidad introdujo criterios adicionales de adjudicación de la capacidad marco, que fueron aprobados por la $\mathrm{CNMC}^{17}$,

\footnotetext{
17 Resolución sobre los criterios de adjudicación de capacidad marco incluidos en la versión definitiva de la modificación de la declaración sobre la red 2019 de las entidades públicas empresariales Administrador de Infraestructuras y Adif-Alta velocidad.

https://www.cnmc.es/sites/default/files/2731113_2.pdf
}

en caso de que se produjera igualdad entre varios candidatos: i) compromisos de reducción de la huella de carbono, ii) menor porcentaje de contratos temporales, iii) mayor porcentaje de mujeres empleadas, $y$, iv) mayor porcentaje de personal con discapacidad.

Las empresas interesadas en prestar sus servicios a partir del 14 de diciembre de 2020 solicitaron capacidad de acuerdo con sus planes de negocio antes del 31 de octubre de 2019. Seis candidatos solicitaron capacidad marco: Renfe, Rielsfera (filial de SNCF), ILSA (Airnostrum con compromiso de participación de Trenltalia), Motion Rail (filial de Talgo), ECORAIL y EAGLE (participada por Globalvía).

Una vez recibidas las solicitudes, ADIF Alta Velocidad constató que el conjunto de peticiones superaba con mucho la capacidad disponible y hacía imposible el proceso de coordinación previsto para hacerlas compatibles. En el segundo año de apertura del mercado, la capacidad media solicitada ya alcanzaría prácticamente la ofertada, siendo incluso un $10 \%$ superior en el caso del corredor Madrid-Barcelona. A partir del horario de servicio 2025-2026, cuando los planes de negocio de los candidatos estuvieran plenamente operativos, la capacidad media solicitada superaría la disponible en un $40 \%$, incrementándose hasta el $70 \%$ en el corredor Madrid-Barcelona.

Dada esta situación, el gestor procedió a aplicar los criterios de priorización establecidos en la Declaración sobre la Red. De forma preliminar, ADIF Alta Velocidad valoró si las solicitudes de capacidad resultaban realistas, constatando la solvencia técnica y económica de las mismas, si bien, en el caso de ILSA, se vinculaba a la participación de Trenltalia en su accionariado.

El 27 de noviembre de 2019, el Consejo de Administración de ADIF Alta Velocidad $\triangleright$ 
acordó asignar provisionalmente la capacidad marco a Renfe (Paquete A), ILSA (Paquete B) y Rielsfera (Paquete $C$ ).

A partir de esta decisión, el gestor de infraestructuras y las empresas adjudicatarias de capacidad marco debían coordinar las solicitudes, incluyendo los aspectos operativos de las mismas, como las paradas intermedias en el trayecto o las prolongaciones a otras estaciones, y firmar el acuerdo marco correspondiente.

\section{Aprobación de los acuerdos marco por la CNMC}

En marzo de 2020, ADIF Alta Velocidad envió a la CNMC las propuestas de acuerdos marco con Renfe, ILSA y Rielsfera. La CNMC analizó en sus Resoluciones de 6 de abril de 2020 tanto el procedimiento seguido por ADIF Alta Velocidad para la adjudicación de la capacidad marco como las propuestas de acuerdos $^{18}$.

El análisis de la información aportada constató que si bien las peticiones de capacidad eran realistas, no todas afrontaban los mismos riesgos. En particular, si bien ADIF Alta Velocidad concluyó que todos los candidatos contarían con material rodante en la fecha de inicio comprometida de las operaciones, la CNMC identificó riesgos de retraso en aquellos casos en los que los trenes debían fabricarse. La compra, producción y homologación de los trenes de viajeros es un largo proceso que oscila entre los tres y cuatro años ${ }^{19}$.

\footnotetext{
18 https://www.cnmc.es/sites/default/files/2906442_9.pdf https://www.cnmc.es/sites/default/files/2906451_0.pdf https://www.cnmc.es/sites/default/files/2906461_2.pdf

19 La incorporación de los trenes AVRIL a la flota de Renfe se inició con la adjudicación por su Consejo de Administración el 28 de noviembre de 2016. Renfe prevé que los primeros trenes de la serie 106 estén disponibles para operar en 2021, una vez que pasen las preceptivas pruebas para su autorización de puesta en servicio por parte de la Agencia Estatal de Seguridad Ferroviaria, esto es, cuatro años después de la formalización de la compra.
}

Los planes de negocio de algunos candidatos prevén el inicio de la prestación de los servicios en un periodo muy inferior, por lo que los riesgos de incumplimiento son superiores a los de otros que, o bien ya cuentan con el material rodante, como Renfe o Rielsfera, que prestará sus servicios con trenes que actualmente están circulando en servicios internacionales entre España y Francia, o circulan en la red francesa; o ILSA, cuyos trenes ya se están fabricando sobre la base de un pedido previo de Trenltalia.

Por ello, la CNMC concluyó que ADIF Alta Velocidad había considerado las mismas variables y criterios para valorar las solicitudes de todos los candidatos, siendo las propuestas más realistas en cuanto a la disponibilidad de material rodante y solvencia financiera las de los tres candidatos adjudicatarios de capacidad marco.

En cuanto a las propuestas de acuerdos marco, la CNMC comprobó que su articulado contara con las previsiones mínimas incluidas en el Reglamento de Ejecución 2016/545, en particular: i) la posibilidad de modificar la capacidad marco solicitada anualmente en un determinado porcentaje (10\%); ii) las penalizaciones aplicables en caso de incumplimiento de los compromisos adquiridos, si bien señalando que cuando el acuerdo marco se modifique por causa de terceros y se comunique al gestor, estas no serán de aplicación; y iii) la vigencia de los acuerdos marco.

En relación con este último aspecto, la Ley del Sector Ferroviario establece que duraciones de los acuerdos marco superiores a cinco años deben ser excepcionales, estar justificadas y acreditadas en el expediente sobre la base de: i) contratos comerciales, ii) inversiones especializadas o iii) riesgos.

En las Resoluciones de 6 de abril de 2020, la CNMC concluyó que ADIF Alta $\square$ 
Velocidad había justificado adecuadamente la extensión a diez años de la vigencia de los acuerdos marco de ILSA y Rielsfera, dadas las inversiones que debían asumir para acceder al mercado español, tanto en términos de material rodante como otros derivados de la red comercial y de operaciones necesarias para permitir la entrada en el mercado español. Por el contrario, en el caso de Renfe, se requirió información adicional dado que, inicialmente, se aportó el material rodante y las inversiones de este candidato para el conjunto de su negocio ferroviario de alta velocidad y no los vinculados a los corredores objeto del acuerdo marco. Además, la antigüedad media de los trenes de Renfe mostraba un riesgo diferencial con respecto a los otros dos candidatos.

Posteriormente, ADIF Alta Velocidad envió información adicional, incluyendo un escrito de Renfe en el que se desagregaba su plan de negocio para la explotación de la capacidad marco objeto del acuerdo. Estas clarificaciones permitieron que la CNMC aprobara su acuerdo marco el 6 de mayo de $2020^{20}$, por una duración de diez años.

Una vez aprobados, todos los acuerdos marco se firmaron el 13 de mayo de 2020.

\subsection{Resultado del proceso de adjudicación de capacidad marco}

La experiencia europea muestra que la liberalización del transporte de viajeros por ferrocarril ha incrementado de forma sensible el número de pasajeros. Por ejemplo, en el periodo 2012-2015, en Italia se incrementó en un 65\% el número de pasajeros, un $91 \%$ en la República Checa y un $25 \%$ en Austria. Este proceso

20 https://www.cnmc.es/sites/default/files/2936034_7.pdf también ha supuesto una mayor actividad en la red ferroviaria. En Italia, el operador alternativo, NTV, ha incrementado las frecuencias a la vez que ha creado nuevas conexiones, alcanzando una cuota del $26 \%$ del mercado nacional en 2016. Esta misma dinámica se observa en Austria, donde la entrada de WestBahn, el operador alternativo, ha permitido aumentar las frecuencias existentes en el corredor Salzburgo-Viena, o en la República Checa, donde la competencia ha llevado a un aumento muy significativo de las frecuencias en la línea Praga-Ostrava ${ }^{21}$.

El resultado del proceso de adjudicación de capacidad marco hace prever una dinámica similar, tanto por el número de surcos adjudicados (+52\% como se observa en el Gráfico 1) como por el número de participantes.

La adjudicación de capacidad marco señala que los beneficios de la competencia se extenderán por los tres principales corredores de alta velocidad en España, evitando un proceso de descreme de mercado que hubiera concentrando la entrada en el corredor entre Madrid y Barcelona.

Dado el incremento de capacidad adjudicada con respecto a la situación actual, es previsible que los precios de los servicios ferroviarios se reduzcan, como ha sucedido en otros países europeos, para atraer viajeros de otros modos de transporte. En este sentido es preciso señalar que, salvo en el trayecto Madrid-Barcelona, donde el avión conserva en torno al $35 \%$ de la cuota modal, en el resto de líneas el ferrocarril ya aglutina la práctica totalidad de los viajeros. Por tanto, el crecimiento de este modo de transporte deberá provenir bien de nuevos tráficos $\triangleright$

21 Finger, M., Kupfer, D., \& Montero-Pascual, J. J. (2016). Competition in the railway passenger market. Florence School of Regulation (FSR). European University Institute. 

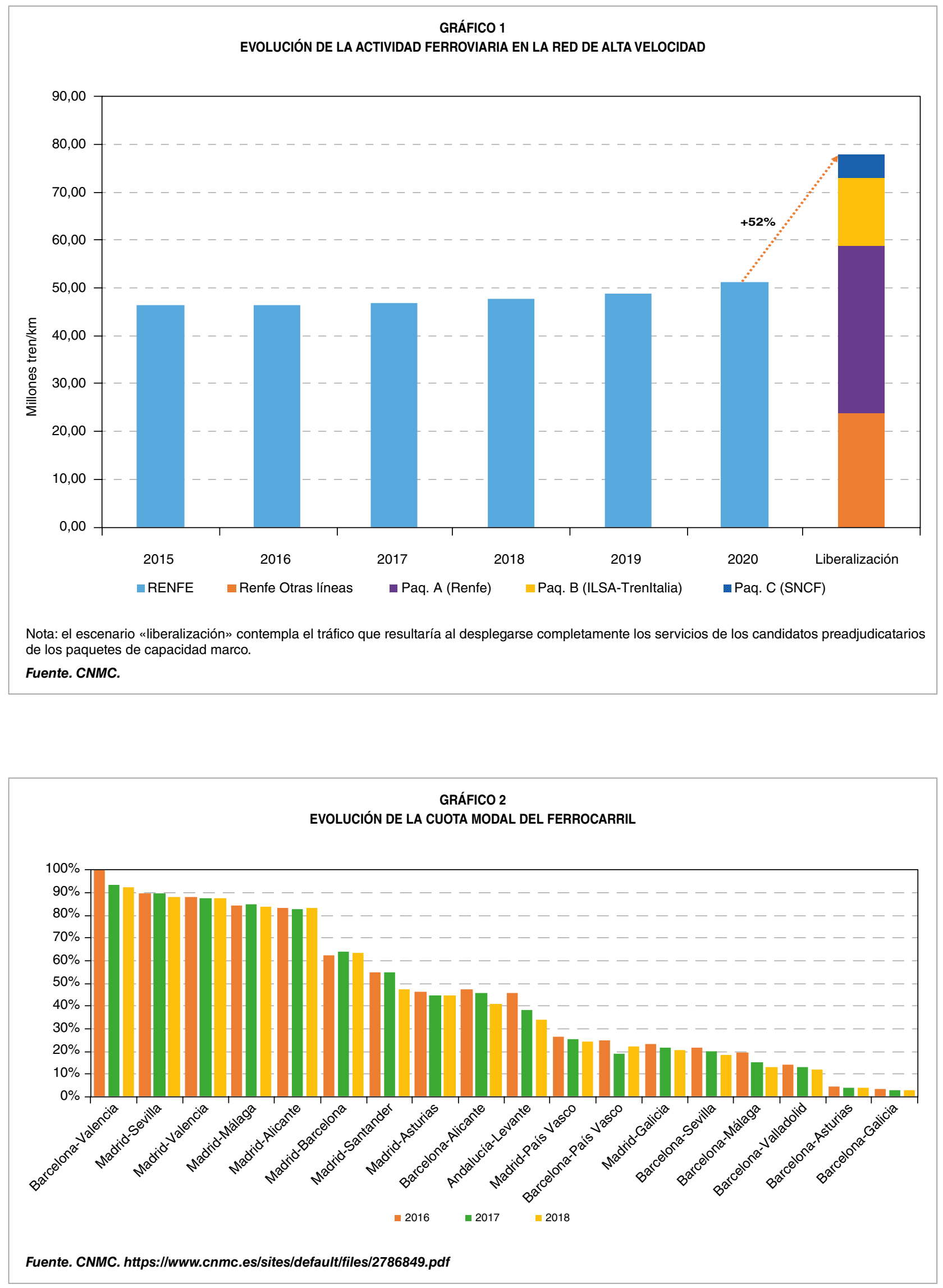
inducidos, bien del trasvase modal desde el coche privado.

El modelo de adjudicación de la capacidad marco elegido por ADIF Alta Velocidad ha sido objeto de debate por su novedoso diseño con respecto a la dinámica de adjudicación de capacidad que impera en el sector ferroviario. En un proceso de solicitud de capacidad ordinario las empresas ferroviarias realizan sus solicitudes, que son analizadas, conjuntamente, por el administrador de infraestructuras. Este adjudica los surcos según las solicitudes y, en caso de detectarse incompatibilidades, las intenta coordinar proponiendo modificaciones para dar satisfacción a todas las solicitudes.

ADIF Alta Velocidad detectó un elevado interés en la liberalización del mercado de viajeros, por lo que incluyó los paquetes de capacidad marco y la priorización en función del uso que se ha descrito anteriormente. Con este diseño hay quien ha considerado que el gestor de infraestructuras dirigía excesivamente los planes de entrada de los candidatos, estableciendo ex ante el tamaño de los paquetes, tiempos de rotación de los trenes con surcos concatenados, duración de los acuerdos y extensión de la entrada a los tres corredores incluidos en la oferta de capacidad marco.

Además, de acuerdo con este argumento, el propio sistema habría incentivado las peticiones de capacidad de los candidatos, convirtiéndose en una especie de profecía autocumplida en la que, dado que ADIF Alta Velocidad previó una demanda de capacidad por encima de la disponible, estableció un sistema de priorización que incentivaba el uso, forzando a los candidatos a solicitar capacidad por encima de la que inicialmente tenían prevista.

La apertura de un mercado es un proceso complejo, en particular, en un sector como el ferroviario, con elevadas barreras a la entrada dadas las inversiones necesarias para operar y los costes hundidos que suponen. Además, este sector adolece de restricciones a la capacidad que impiden una apertura similar a la de otros mercados, como en las telecomunicaciones, en los que se produjo la entrada de un elevado número de empresas para, posteriormente, consolidarse en un número más reducido capaz de alcanzar economías de escala.

Las limitaciones en determinadas infraestructuras impiden la competencia de un número elevado de empresas, por lo que es necesario establecer criterios de priorización ex ante. En este contexto debe entenderse la iniciativa de ADIF Alta Velocidad, dado que, además, la regulación de la asignación de capacidad no cuenta con reglas concretas, como en la aviación, que aseguran, por ejemplo, slots en aeropuertos a las aerolíneas de bandera o reservan una parte de la capacidad a nuevos entrantes.

Una vez fijada la fecha de apertura del mercado mediante el Real Decreto Ley 23/2018, el gestor de infraestructuras debía atender las solicitudes de capacidad de cualquier candidato. Dada la falta de una regulación específica en la asignación de capacidad, a priori, la regla de asignación aplicable sería «primero en pedir capacidad, primero en ser atendido», teniendo una ventaja clara aquellos candidatos que ya contaban con trenes.

En este escenario, además, la entrada se concentraría en el corredor Madrid-Barcelona, que aglutina una parte sustancial del tráfico de alta velocidad. Acaparar la capacidad de este corredor tiene el riesgo de evitar la competencia en el resto de la red, limitando su optimización y los beneficios para una parte sustancial de la población.

Por tanto, un candidato con material rodante podría haber solicitado capacidad cuando $D$ 
el mercado se liberalizara, debiendo ser atendida por ADIF Alta Velocidad. Esta situación suponía el riesgo de desincentivar la entrada de otros entrantes si se concentra en los trayectos más atractivos. En caso de que las mismas empresas que han participado en el proceso de liberalización hubieran mostrado su interés, el gestor habría afrontado una importante sobredemanda sin unas reglas ex ante claras de priorización.

De esta forma, es claro que la anticipación de ADIF Alta Velocidad en el diseño de unas reglas claras de asignación de capacidad ha aportado transparencia al proceso y limitado su conflictividad.

El sistema de solicitud de capacidad ordinaria del horario anual tampoco es un referente válido para este proceso. La asignación anual se realiza surco a surco, siendo el gestor el que debe coordinarlos uno a uno. En el caso de los servicios de alta velocidad, los surcos deben tener cierta coherencia, de forma que se maximice el uso de los trenes. Sin la concatenación de los surcos se corre el riesgo de esperas del material rodante en las estaciones terminales de los trayectos hasta el siguiente surco disponible, incrementando los costes de la operación.

Por este motivo, la agrupación de capacidad en paquetes de surcos concatenados, incluida por ADIF Alta Velocidad en la Declaración sobre la Red, responde a la maximización del uso del material rodante.

La coordinación de las peticiones de capacidad es un proceso complejo que, si bien puede suponer cambios menores en los surcos (hora de salida o en paradas intermedias), con un importante exceso de peticiones, el resultado puede determinar el modelo de negocio de los candidatos. En concreto, en el caso de los tres corredores objeto de adjudicación de capacidad marco, el exceso de demanda suponía que la coordinación hubiera desnaturalizado algunos modelos de negocio, en particular los basados en una competencia en frecuencias para captar el tráfico de negocios, e incluso poner en cuestión la mínima masa crítica para hacer rentable el propio modelo.

Finalmente, como se constató en las resoluciones de la CNMC sobre la aprobación de los acuerdos marco, las inversiones necesarias para competir en el mercado de transporte de viajeros por ferrocarril son elevadas. Por tanto, con independencia del diseño de la oferta de capacidad marco, que efectivamente establecía como criterio de adjudicación el mayor uso de la misma, es improbable que los candidatos incrementaran artificialmente las solicitudes de capacidad marco para asegurarse la entrada. Además de las inversiones señaladas, los acuerdos marco incluían penalizaciones por el incumplimiento de los compromisos asumidos en los acuerdos marco con el objeto de incentivar peticiones de capacidad realistas.

A la vista de los riesgos y costes asociados a peticiones no realistas, es muy discutible que el diseño de la oferta de capacidad marco haya sido el causante de las elevadas solicitudes de los candidatos. Por el contrario, resulta más plausible que el proceso haya despertado un elevado interés que haya debido ser administrado dando transparencia ex ante a los criterios de adjudicación.

En definitiva, la oferta de capacidad marco diseñada por ADIF Alta Velocidad fue novedosa y vinculaba su adjudicación a determinadas características del plan de negocio del candidato. En un contexto de exceso de capacidad, una vez introducidas las medidas de flexibilización recomendadas por la CNMC, su diseño habría permitido una optimización de la red ferroviaria en los tres corredores incluidos en el $\triangleright$ 
proceso. Si bien el sistema establecido por el gestor puede haber modificado los planes iniciales de algún candidatos, un análisis de las alternativas existentes de acuerdo con el marco regulador muestra que, o bien el resultado habría sido similar, dado el interés despertado por la liberalización en España, o habría desembocado en una desnaturalización de los modelos de negocio que habría restringido la pluralidad de la oferta o, incluso, cuestionado la viabilidad de algunas de las iniciativas de entrada.

\section{Próximos pasos}

Una vez finalizada la adjudicación y firmados los acuerdos marco, ADIF Alta Velocidad debe ofrecer la capacidad que no fue asignada en la primera fase mediante una actualización de la oferta de capacidad marco. A la vista de los surcos no solicitados, los candidatos podrán solicitar la firma de un acuerdo marco en caso de que dicha capacidad remanente permita ofrecer servicios de forma viable.

A este respecto, es relevante el impacto de la crisis generada por la COVID-19, tanto por los retrasos en las modificaciones del material rodante y su fabricación por el cierre de los centros productivos como por los efectos de la situación económica en la demanda de transporte.

Los acuerdos marco prevén que los compromisos adquiridos por los candidatos no serán de aplicación en una situación de fuerza mayor 22 . Además, el artículo 13.3 del Reglamento 2016/545 establece que las penalizaciones no serán de aplicación «cuando el acuerdo

22 Ver Anexo J de la Declaración sobre la Red de ADIF Alta Velocidad. http://www.adifaltavelocidad.es/es_ES/conocenos/doc/DR_ADIF_AV_ V1_2020_20200604.pdf haya sido modificado o anulado por causas ajenas a la voluntad del candidato y que hayan sido comunicadas sin demora al administrador de infraestructuras».

Tanto los acuerdos marco como la normativa que los regulan prevén mecanismos para hacer frente a la situación creada por la COVID-19, si bien con diferencias relevantes entre ellas. Así, mientras que la aplicación de la cláusula de fuerza mayor no supone la modificación del acuerdo marco y, por tanto, la liberación de la capacidad marco, la aplicación del Reglamento 2016/545 sí supone su actualización y, en su caso, la modificación de la oferta de capacidad marco, permitiendo que aquella que no vaya a ser aprovechada se solicite por terceros.

El gestor de infraestructuras deberá decidir el alcance de las excepciones al cumplimiento del acuerdo marco por razones de fuerza mayor sin exigir su modificación. Desde un punto de vista competitivo, y dado el exceso de peticiones recibidas, sería recomendable modificar los acuerdos marco si se constatara que algún adjudicatario no solicitara de forma continuada la capacidad comprometida, poniéndola a disposición de terceros.

Por otra parte, según el artículo 59 de la Ley del Sector Ferroviario, los candidatos deben comunicar con antelación los servicios que se solapen con itinerarios sujetos a obligaciones de servicio público (OSP). El acceso a la red ferroviaria podría restringirse si pusieran en riesgo el equilibrio económico del contrato de servicio público, algo que debe analizar la CNMC.

Tanto ILSA como Rielsfera han comunicado su intención de prestar servicios en líneas de alta velocidad coincidentes con el servicio AVANT de Renfe, sujeto a OSP ${ }^{23}$. En caso $D$

23 https://www.cnmc.es/ambitos-de-actuacion/transporte/ nuevos-servicios-ferroviarios 
de que se solicite la prueba de equilibrio económico, la CNMC deberá analizar estos servicios de acuerdo con la metodología que aprobó el 16 de julio de $2020^{24}$.

El acceso a las instalaciones de servicio, en particular estaciones de viajeros y talleres de mantenimiento, es un elemento esencial para la liberalización del transporte de viajeros por ferrocarril. En el primer caso, la experiencia italiana muestra las dificultades que pueden afrontar las empresas alternativas para ocupar espacios y dar servicio a sus clientes en igualdad de condiciones con la empresa establecida. De hecho, el regulador hubo de intervenir para asegurar el acceso del nuevo operador (NTV) a las estaciones gestionadas por el administrador de las infraestructuras (RFI), incluyendo espacios para la colocación de máquinas expendedoras de billetes en los vestíbulos de las estaciones o para las salas VIP25. En relación con el mantenimiento de los trenes, NTV optó por construir su propio taller con una inversión aproximada de 90 millones de euros ${ }^{26}$.

En el caso español es necesaria la adaptación a un entorno multioperador de estas instalaciones, diseñadas para prestar servicios a una única empresa. ADIF Alta Velocidad publicó las características de las principales estaciones de alta velocidad, así como los espacios disponibles, su ubicación y criterios de asignación en la Declaración sobre la Red publicada en diciembre de 2019, un año antes de la liberalización del mercado ${ }^{27}$. La CNMC analizó la propuesta del gestor en el Informe de 21

\footnotetext{
24 https://www.cnmc.es/sites/default/files/3058981.pdf

25 Por ejemplo, las operaciones de NTV en la estación de Rimini (Italia) han sido objeto de conflicto con RFI por el retraso de varios meses en las obras necesarias para construir un andén para que operen los trenes de alta velocidad por parte de RFI (Trepat, 2018).

26 International Railway Journal (14 de diciembre de 2011).

27 http://www.adifaltavelocidad.es/es_ES/conocenos/doc/DR_ADIF_ AV_V1_2020_20200604.pdf
}

de noviembre de $2019^{28}$, valorando de forma positiva la información publicada y la consideración de criterios cualitativos de representatividad y protagonismo en la distribución de los espacios de venta de billetes en las estaciones, si bien se consideró que estos criterios debían también aplicarse a otros espacios, como las salas VIP.

Renfe Mantenimiento, titular de la mayor parte de las instalaciones de mantenimiento y en particular las relacionadas con los trenes de pasajeros, también publicó su oferta de servicios en la Declaración sobre la Red. De acuerdo con la oferta publicada, esta empresa ofrece a terceros todas las operaciones que supongan estadías de hasta siete días en el taller. A este respecto es necesario destacar la proactividad de esta empresa en ofrecer sus servicios a terceros, lo que sin duda facilitará la entrada de empresas en el mercado de prestación de servicios de viajeros, dados los plazos que supone la construcción de un taller propio.

Finalmente, la regulación de los cánones ferroviarios es un elemento fundamental para las empresas ferroviarias dado su peso en los costes totales, que pueden alcanzar el $45 \%$ en el caso de los servicios de alta velocidad. A este respecto, la CNMC informó una propuesta de Anteproyecto de Ley del Ministerio de Transportes, Movilidad y Agenda Urbana (MITMA) por el que se modifica el sistema de aprobación de los cánones ferroviarios ${ }^{29}$. Actualmente, este proceso se inicia con una propuesta del gestor de infraestructuras que es sometida a consulta de las empresas ferroviarias, que es supervisada por la CNMC y finalmente comunicada al MITMA para su incorporación en la Ley de Presupuestos Generales de Estado.

\footnotetext{
28 https://www.cnmc.es/sites/default/files/2758165.pdf

29 https://www.cnmc.es/sites/default/files/2920257.pdf
} 
Tanto la CNMC como la Comisión Europea han cuestionado este modelo porque podría incumplir los requisitos de independencia de los gestores de infraestructuras establecidos en el marco europeo. Además, en los últimos años, la aprobación de los Presupuestos Generales del Estado no se ha producido anualmente, lo que ha retrasado la aplicación de las modificaciones propuestas por los gestores de infraestructuras, en particular, aquellas que orientaban los cánones a los costes directos.

La nueva propuesta del MITMA permitirá que ADIF y ADIF Alta Velocidad aprueben los cánones mediante su publicación en la Declaración sobre la Red. Gracias a esta modificación, los cánones ferroviarios deberían convertirse en una herramienta de gestión de la red ferroviaria que incentive su uso.

\section{Conclusiones}

La liberalización de los servicios de transporte de pasajeros culmina un largo proceso que se inició en 2005 con la liberalización de los servicios de mercancías. La red ferroviaria de alta velocidad cuenta con características que facilitan la entrada de nuevas empresas, como son su bajo nivel de congestión y su independencia de los servicios más intensivos en capacidad, como, por ejemplo, las cercanías de los grandes núcleos de población. Esta situación explica el interés despertado por el proceso, en el que han participado seis empresas, un escenario inédito en Europa.

De acuerdo con la experiencia de otros países europeos, la apertura de estos servicios puede reportar importantes beneficios para los usuarios, el gestor de las infraestructuras y la sostenibilidad del transporte en España. Las peticiones de capacidad indican que la actividad ferroviaria se incrementará sustancialmente, lo que contribuirá a rentabilizar las cuantiosas inversiones realizadas en la red ferroviaria de alta velocidad. Además, el previsible incremento del tráfico facilitará la sostenibilidad financiera de ADIF Alta Velocidad, que afronta el proceso con una deuda de más de 16.000 millones de euros. Finalmente, la competencia entre las diferentes empresas puede propiciar el cambio modal al ferrocarril, un medio más sostenible que otros, como el coche privado.

La participación en el mercado de las empresas ferroviarias requiere acceder a la red ferroviaria. ADIF Alta Velocidad abordó este aspecto dando transparencia a la capacidad disponible y proponiendo un sistema de asignación relativamente novedoso, basado en paquetes de surcos concatenados en los tres principales corredores de alta velocidad, que se adjudicarían al candidato que se comprometiera a un mayor uso en un periodo de diez años.

Si bien este esquema no se ajusta al estándar que impera en el sector ferroviario, ha resultado útil para asegurar una transición ordenada, garantizando que no se produzca una discontinuidad en la prestación de los servicios ferroviarios. Además, asegura una competencia entre tres modelos de negocio muy diferentes y con un incremento de más del $50 \%$ con respecto a las frecuencias actuales.

El proceso de apertura requiere, además de la capacidad en la red, el acceso a otras instalaciones ferroviarias, como las estaciones de viajeros y talleres de mantenimiento. A este respecto es necesario destacar que los principales explotadores de las mismas ya han publicado las condiciones de acceso, permitiendo que los candidatos adjudicatarios de capacidad conozcan las condiciones en las que $D$ 
Iván Santos Esteras

podrán prestar sus servicios en las estaciones o mantener sus trenes.

Finalmente, en relación con una de las principales partidas de costes de las empresas, los cánones ferroviarios, se está modificando la Ley del Sector Ferroviario para desvincularlos de los Presupuestos Generales de Estado. Esta propuesta debería convertir los cánones ferroviarios en una herramienta de gestión de la red y no en una figura tributaria como hasta la fecha. 